\title{
Griffonia simplicifolia I: Fluorescent Tracer for Microcirculatory Vessels in Nonperfused Thin Muscles and Sectioned Muscle
}

\author{
F. M. Hansen-Smith, ${ }^{1}$ L. Watson, D. Y. Lu, ${ }^{2}$ and I. Goldstein \\ Department of Biological Sciences, Oakland University, Rochester, Michigan 48309-4401, and \\ Departments of Anatomy and Cell Biology, and Biological Chemistry, University of \\ Michigan, Ann Arbor, Michigan 48109
}

Received April 2, 1987

\begin{abstract}
Previous studies on mice have revealed that the Griffonia simplicifolia I (GSI) lectin selectively binds to capillaries in a number of microvascular beds. These observations suggest that the lectin might be a suitable microvascular marker for physiological studies of skeletal muscle, particularly when fluorescent visualization of vessels is desired independently of their perfusion status. Since species and strain heterogeneity has been demonstrated for certain lectins associated with the microcirculatory vessels, lectin binding was studied in a number of muscles taken from the major species of mammals used for experimental purposes. Staining of cryostat sections confirmed the utility of GSI as a marker for capillaries from muscle of mice, rats, hamsters, rabbits, dogs, and monkeys. Differential staining of arterioles and veins was revealed by double labeling with GSI and antisera to Factor VIII-related antigen. Double labeling for GSI binding and alkaline phosphatase activity revealed that the GSI method detects many more capillaries and terminal arterioles than does the alkaline phosphatase method. GSI binding to unfixed whole mounts of thin skeletal muscles (hamster cheek pouch, mouse diaphragm, and rat cremaster) was studied to determine whether the GSI lectin would be a suitable marker for intravital studies. An extensive microvascular bed, including terminal arterioles, venules, and capillaries, was revealed which could be visualized in the complete absence of perfusion with fluorescent markers. These observations suggest that the GSI lectin may be extremely useful as a probe for the microcirculation of skeletal muscle in many types of physiological experiments.

() 1988 Academic Press, Inc.
\end{abstract}

The microvascular beds of skeletal muscle are dynamic systems which change in true dimension and geometry in response to various acute and chronic physiological conditions (Hudlicka, 1982). The dimensions and geometry of the perfused microvascular bed are also subject to marked changes in response to pharmacological agents. There are only a few muscles in mammals which are thin enough to be used for direct intravital studies of these changes. Current technology makes it difficult to relate observations of the perfused microvascular bed to the whole microvascular bed in such preparations. Until now, it has been impossible to visualize the terminal vessels and capillaries unless they are perfused (Damon and Duling, 1984). For studies of the microcirculation of larger muscles it has

\footnotetext{
' To whom reprint requests should be addressed.

${ }^{2}$ Present address: National Institute of Sports Science, Histopathological Laboratory, Beijing, China.
} 
been necessary to use histological methods to visualize the microvascular bed, with a reliance on the presence of opaque markers such as India ink or casting materials to show the microvascular geometry (Schraufnagel et al., 1983; Van Reempts et al., 1983; Potter and Groom, 1983). Capillaries have been identified cytochemically by using histochemical stains for enzymes such as alkaline phosphatase (APase) or ATPase, sometimes in combination with PAS or diaminopeptidase to reveal more of the terminal vessels (Romanul and Bannister, 1962; Plyley and Groom, 1975; Sillau and Banchero, 1977; Maxwell et al., 1980; Lipenska and Horsky, 1984; Ogilvie and DeHay, 1986). However, such histochemical procedures cannot be applied to reveal the unperfused microvascular bed in intravital studies.

One of the most valuable histochemical techniques to emerge in recent years has been the use of lectins to probe the terminal saccharides associated with cell surfaces (McCoy, 1986). Lectins are proteins, usually derived from plants, which show selective, reversible binding to terminal saccharides. For histochemical purposes the lectins can be used with a visible marker, such as the peroxidase derivatives, or with fluorescent markers, such as the fluorescein or rhodamine derivatives. The Ulex lectin was shown to selectively bind to endothelial cells in human tissucs. However, this lectin binding is apparently unique to human tissue and is therefore not suitable for the use with animal models (Holthofer et al., 1982; Holthofer, 1983; Hansen-Smith and McCoy, unpublished). Some of the galactose-binding lectins have been noted to have an affinity for endothelial cells (Peters and Goldstein, 1979; Gerhart et al., 1986, Hansen-Smith, 1985). Ponder and Wilkinson reported a specificity of Dolichos biflorus agglutinin for vascular endothelium in mice, but both organ-related specificity and strain specificity were demonstrated by these investigators (Ponder and Wilkinson, 1983; Ponder et al., 1985). Species specificity of certain lectins has also been reported (Holthofer et al., 1982; Ribera et al., 1987).

The purpose of the present study was to evaluate the use of a specific lectin, Griffonia simplicifolia I (GSI, formerly known as Bandeiraea simplicifolia, BSI), as a marker for capillaries and the larger microcirculatory vessels, in mammalian skeletal muscle. Peters and Goldstein (1979) first reported the selective binding of the GSI $\mathrm{B}_{4}$ isolcctin to capillarics in mousc skcletal muscles. Laitinen (1987) recently repeated these observations, using tissues from a number of unspecified strains of mice. Because of the uncertainty regarding species and strain specificity for this lectin, our first goal was to determine whether the GSI-B 4 lectin would be a suitable microvascular marker for experimental models commonly used for microvascular research. We therefore examined the binding of GSI-B B $_{4}$ in a number of skeletal muscles from several laboratory mammals.

Despite previous reports noting the binding of GSI to capillaries in muscle and many other tissues, these observations have not been applied to questions related to the physiology of the microvascular bed in skeletal muscle. Information is lacking concerning the extent of the lectin binding within the microvascular bed. For example, the specific types of blood vessels (other than capillaries) that bind the lectin have not been identified. Furthermore, there is no information concerning whether all capillaries bind the GSI lectin or whether some capillaries or other terminal vessels fail to express the characteristic, as is the case for alkaline phosphatase activity (Romanul and Bannister, 1962; Mrazkkokova et 
al., 1986; Ogilvie and DeHay, 1986). We therefore undertook an extensive examination of the specific microvascular structures that bind the GSI lectin in sectioned skeletal muscles from rats. In order to evaluate the potential of the GSI lectin for intravital physiological studies, we also examined GSI lectin binding in whole mounts of thin muscles from mice, hamsters, and rats. The distribution of GSI binding sites was compared with that of two other microvascular markers, Factor VIII-related antigen (VIII-R) and APase activity. The results reveal many advantages in the use of this lectin for physiological studies of thin, intravital preparations as well as for sectioned muscle, using either fluorescent or transmitted light microscopic methods.

\section{METHODS}

Lectins. Two forms of the GSI lectin were used: The $\mathrm{B}_{4}$ isomer of the GSI lectin, which has a specific affinity for $\alpha$-methyl-D-galactopyranosyl ( $\alpha$-gal) groups, and the less specific GSI lectin, which has an affinity for $N$-acetylgalactosamine in addition to $\alpha$-gal. Fluorescent derivatives of the GSI lectin were obtained from E-Y Labs, Cappel, and Sigma. Both FITC (fluorescein isothyocyanate) and TRITC (rhodamine tetramethylisothyocyanate) derviatives of GSI were used as fluorescent probes. The biotinylated derivative of GSI was used in combination with avidinTRITC for fluorescence or avidin-peroxidase (E-Y Labs) for transmitted light microscopic studies. The $\mathrm{B}_{4}$ isomer of the GSI lectin was isolated from $G$. simplicifolia seeds as previously described (Murphy and Goldstein, 1977). The lectin was conjugated with FITC by the method of Peters and Goldstein (1979).

Specimens. Muscles for cryostat sectioning were obtained from mature SpragueDawley rats, 129svS mice, and Golden Syrian hamsters of both sexes. The animals were sacrificed by an overdose of ether or by exposure to $\mathrm{CO}_{2}$ immediately before the muscles of interest were removed. Muscles from New Zealand rabbits, mongrel dogs, and a Rhesus monkey were removed as biopsies during the course of surgical procedures that were otherwise unrelated to the present study. The specific muscles used are listed in Table 1.

The muscles or muscle samples were removed, rinsed in physiological saline, and quick-frozen in a slurry of isopentane $/ \mathrm{CO}_{2}$. Cryostat sections of transversely oriented skeletal muscle were collected on glass coverslips. Most of the tissues were sectioned at a thickness of $4-$ to $6-\mu \mathrm{m}$, but sections up to $12 \mu \mathrm{m}$ were found to give satisfactory results. The sections were allowed to air-dry overnight and were then either exposed to the lectin the following day or stored at $-20^{\circ}$ or $-70^{\circ}$ for up to 12 months prior to use. Paired sections treated immediately yielded identical results to sections stored at $-20^{\circ}$ or $-70^{\circ}$.

Lectin binding to cryostat sections. The following protocol was followed for the exposure of the frozen sections to the fluorescent lectins:

(1) A containment ring of rubber cement was formed around the section, using a syringe to dispense the cement. This procedure allowed us to minimize the quantity of lectin that was required (usually $10-15 \mu \mathrm{l} / \mathrm{section}$ ).

(2) Sections were preincubated in physiologically buffered saline (PBS) at room temperature for $10 \mathrm{~min}$. The solution was then lightly blotted off by drawing filter paper across the ring.

(3) Sections were exposed to the fluorescent lectin $(25 \mu \mathrm{g} / \mathrm{ml}$, diluted with 
PBS) for $30 \mathrm{~min}$. The coverslips were kept in a dark, moist container at room temperature during this procedure.

(4) Sections were rinsed twice in PBS for $1 \mathrm{~min}$ each, then mounted on slides with the water-soluble mountant, Gelvatol (Monsanto). The sections were dried in the dark overnight prior to examination. If the sections could not be examined immediately, they were kept covered in the refrigerator with no loss of fluorescence. For prolonged storage, slides were kept in a freezer to prevent deterioration.

Controls. One or two coverslips in each batch were exposed to the hapten inhibitor, 0.1-0.01 $M$ methyl- $\alpha$-D-galactosyl pyranoside (Pfansteihl) concurrently

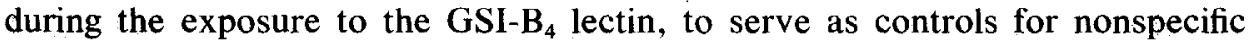
fluorescence.

Modifications of procedures for biotinylated lectins. The biotinylated GSI was applied to the cryostat sections for $30 \mathrm{~min}$. The avidin derivative (TRITC or peroxidase) was added after two 10 -min rinses with $0.1 M \mathrm{NaHCO}_{3}$. After a 20min incubation with the avidin derivative, the sections were again rinsed with $\mathrm{NaHCO}_{3}$ and either mounted (fluorescence) or developed according to the DAB procedure for transmitted light microscopy. (Graham and Karnovsky, 1966).

Lectin binding in thin muscles. The utility of the lectin binding in thin muscles was tested in whole mounts. The mouse diaphragm, hamster cheek pouch, and rat or hamster cremaster muscles were removed and rinsed in PBS. The muscles were then immersed in the fluorescent lectins for $30 \mathrm{~min}$, with occasional agitation. They were then rinsed twice in PBS and mounted on slides, using Gelvatol as a mountant. In order to facilitate the spreading of the tissues on the slides, it was necessary to cut the muscles into smaller pieces.

Double-labeling procedures for cryostat sections. We compared the binding of the lectins with two other markers for endothelial cells: Factor VIII-R and APase activity. Double immunostaining was achieved by first exposing $6-\mu \mathrm{m}$ sections to TRITC-GSI, as described above. This was followed by rabbit antiserum against human VIII-R antigen (Cappell) and FITC-goot anti-rabbit serum. For the enzyme localization, freshly cut $12-\mu \mathrm{m}$ sections were first exposed to TRITCGSI. They were then rinsed in PBS, fixed in acetone, and reacted according to the procedure of Maxwell et al., 1980. Whenever this latter double-staining procedure was carried out, adjacent sections were also processed individually for either the GSI binding or the APase.

Photography. The sections were examined with either a Leitz-Orthoplan or a Nikon Optiphot microscope, each equipped with epifluorescence optics. Photomicrographs were made of selected fields, using a photometer to determine the appropriate exposure time for each section. The exposure times varied slightly among species and were different for different magnifications (10-50 $\times$ ). The 40 and $50 \times$ micrographs were made using either glycerol or oil immersion objectives. Kodak Tri-X or Recording film was used for black-and-white photography. ASA settings of 400-1600 were used, depending on the film and the magnification. Identical settings were used whenever photographs relevant to comparisons of the intensity of fluorescence were being made.

Analyses. Measurements of representative microvascular diameters were taken directly from the slides of the tissue sections and whole mounts, using an ocular micrometer. The GSI/APase double-stained sections were analyzed by photographing six fields per muscle via fluorescence and then by transmitted light 
TABLE 1

Distribution of Griffonia simplicifolia-B 4 Isolectin Binding Sites in Microcirculatory Vessels of Skeletal Muscle

\begin{tabular}{lcccc}
\hline & & & Skeletal muscle $^{a}$ & \\
\cline { 3 - 5 } Species & $n$ & Capillaries & Arterioles & Venules \\
\hline Rat & 10 & ++++ & ++ & $+/-$ \\
Mouse & 10 & ++++ & ++ & $+1-$ \\
Hamster & 4 & ++++ & ++ & $+/-$ \\
Rabbit & 3 & ++++ & ++ & $+1-$ \\
Dog & 3 & ++1 & & \\
Monkey & 1 & ++++ & & \\
\hline
\end{tabular}

${ }^{a}$ Muscles examined include rat and mouse: extensor digitorum longus, soleus, gastrocnemius, tibialis anterior, sternohyoid, sternomastoid, diaphragm, cremaster; hamster: extensor digitorum longus, soleus, cheek pouch; rabbit: diaphragm, intercostal, gastrocnemius; dog: intercostal; monkey, intercostal.

microscopy, at an original magnification of $200 \times$. The fields were randomly selected with regard to the staining of the microvascular components. The number of capillaries revealed with each procedure was then tabulated. The results for each muscle were compared by a $t$ test.

\section{RESULTS}

Cryostat sections of skeletal muscles exposed to the GSI-B ${ }_{4}$ lectin revealed similar patterns of fluorescence in the various muscles from the different species examined (Table 1). Fluorescence due to the lectin hinding was completely eliminated when the tissues were exposed to the hapten inhibitor. The only residual fluorescence under these conditions was due to the elastic lamina of arterioles. There were no noticeable differences between the fluorescence pattern of GSI-B ${ }_{4}$ and that of GSI. Therefore, once the species comparisons were made, the experiments were carried out with the less expensive GSI lectin derivatives.

The fluorescence observed in the sections exposed to GSI was almost exclusively due to binding to microvascular structures (Fig. 1). Neither muscle fibers nor nerves bound the GSI in amounts adequate to make them visible by fiuorescence microscopy. However, portions of the tendonous attachments that were sometimes included in the sections bound the GSI. The only other fluorescent structure in the sections was an occasional granular cell, tentatively identified as a residual macrophage, on the basis of other studies with the GSI lectin (Simon et al., 1986).

Several components of the microvascular bed were revealed by the GSI or GSI- $B_{4}$ binding. Circular profiles of capillaries $(3-6 \mu \mathrm{m})$ were distributed around the periphery of the muscle fibers (Figs. 1 and 2). Also positive for GSI were slightly larger profiles $(6-9 \mu \mathrm{m})$, presumably venous capillaries, located around the periphery of the muscle fibers. The endothelium of arterioles (10-18 $\mu \mathrm{min}$, o.d.) and small arteries ( $>20 \mu \mathrm{m}$, o.d.) were also strongly positive for the GSI lectin (Fig. 3). The cell surfaces of the smooth muscle in the wall of these vessels also bound the lectin, although slightly less strongly than the capillary endothelial 


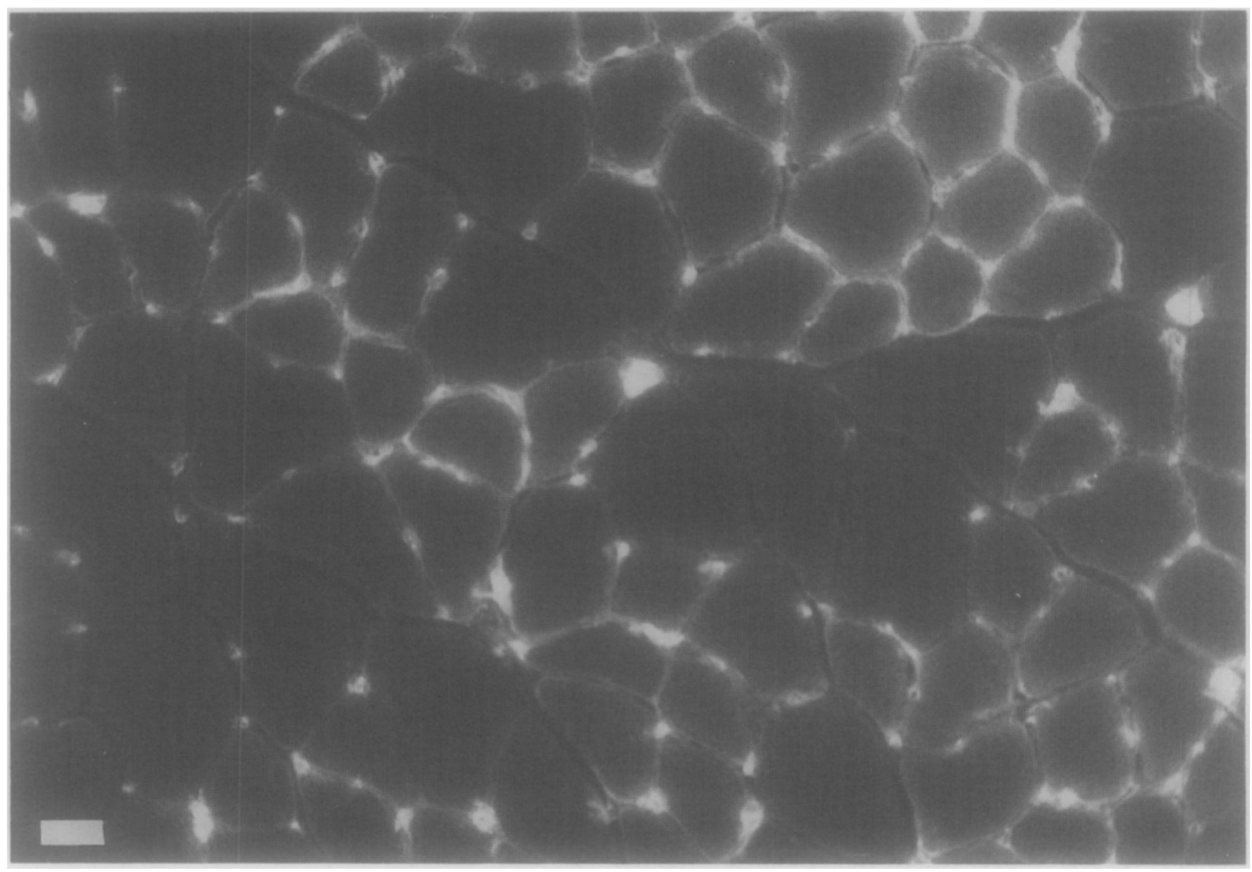

FIG. 1. Fluorescent micrograph shows FITC-GSI-B ${ }_{4}$ lectin binding to capillaries in $10-\mu \mathrm{m}$ section of rat soleus muscle $(\mathrm{Bar}=20 \mu \mathrm{m})$.

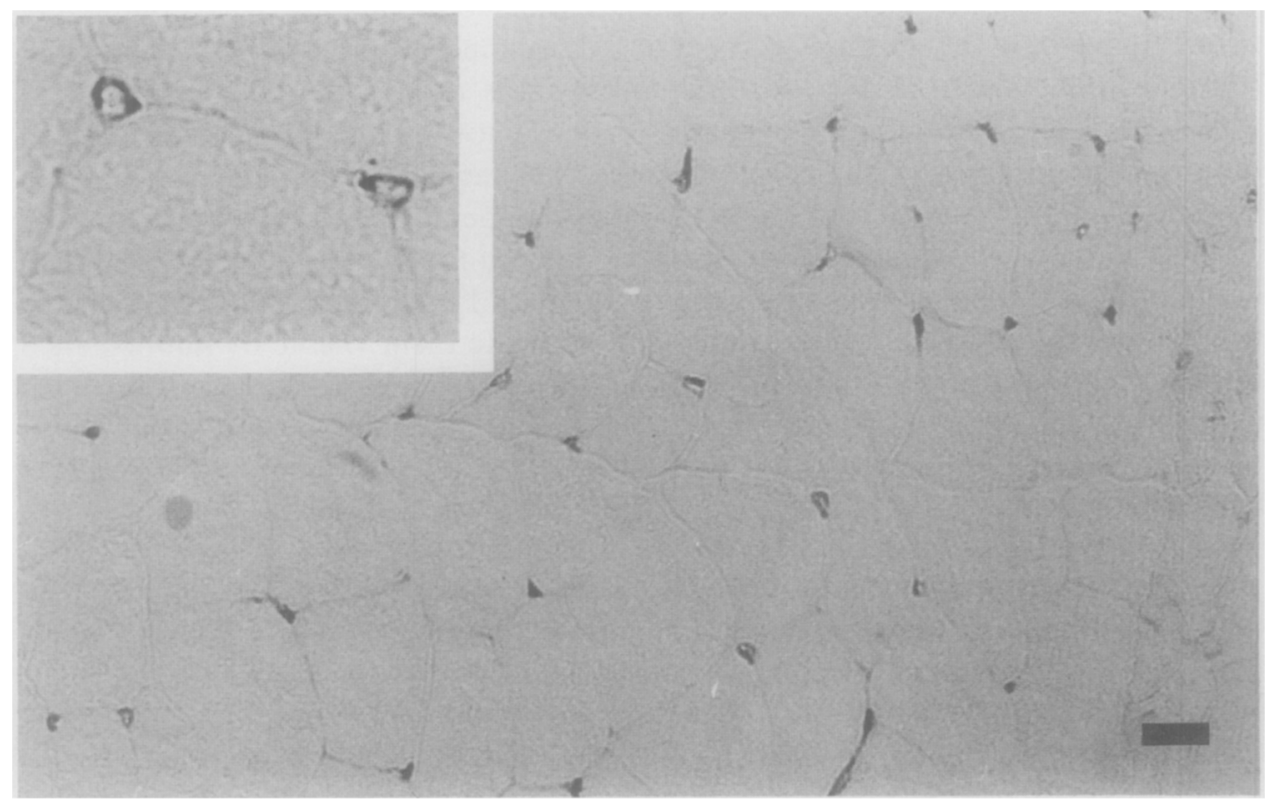

FIG. 2. Rat sternomastoid muscle (6- $\mu \mathrm{m}$ sections) exposed to biotinylated GSI, followed by avidin peroxidase (see text). Inset shows detail of two venulur capillaries. 


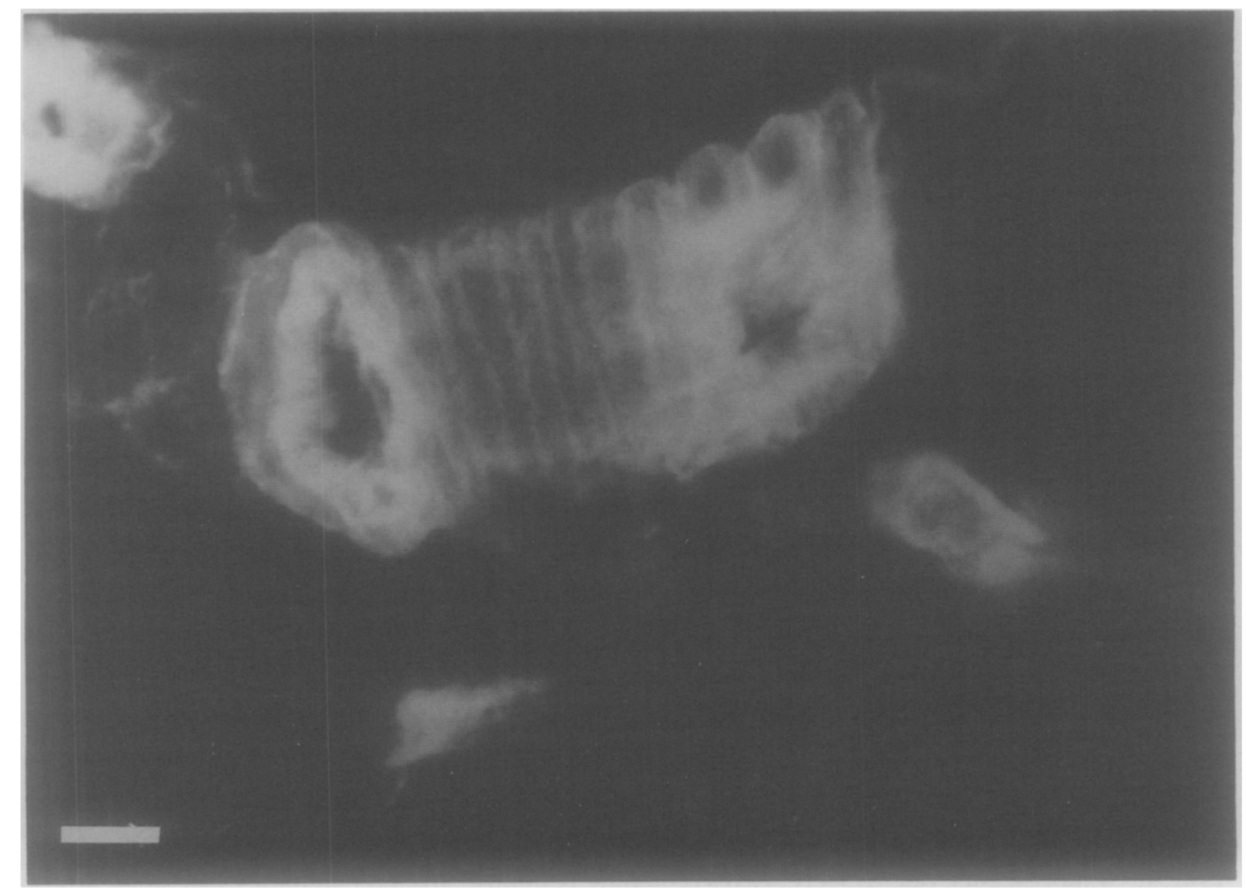

FIG. 3. FITC-GSI-B 4 lectin binding to a longitudinally oriented arteriole from mouse muscle (4$\mu \mathrm{m}$ section) reveals selective binding to endothelium and basal laminae of smooth muscle cells (Bar $=10 \mu \mathrm{m})$.

cells. GSI binding to the endothelium of postcapillary venules $(10-16 \mu \mathrm{m})$ was slightly weaker than that of the capillaries. Smooth muscle walls were not discernable in these vessels. The larger veins within the interfascicular space (i.e., veins that had discernable smooth muscle associated with their walls) revealed only minimal fluorescence (Fig. 4). Weaker fluorescence of veins vs. arteries was found consistently, even when the concentration of the lectin was increased to $100 \mu \mathrm{g} / \mathrm{ml}$, suggesting a lower density of the terminal $\alpha$-gal residues in the venous side of the microvascular bed.

Application of the GSI lectin to the thin muscles revealed extensive fluorescent microvascular beds (Figs. 5-7). The capillary beds were clearly outlined, arranged in parallel around the muscle fibers of the diaphragm and in arcades in the cheek pouch and cremaster muscles. Circularly arranged smooth muscle cells of arterioles (9-22 $\mu \mathrm{m}$ o.d.) could also be detected by the lectin binding, although the smooth muscle in the whole mounts appeared less strongly fluorescent than that in the sectioned muscle. When the course of a vessel was followed from the arteriole, past the capillary bed, a diminution of fluorescence was evident on the venous side. This corresponded to the reduction in fluorescence observed in veins of sectioned muscles. Whereas the terminal branches in these microvascular beds bound the GSI, the largest arteries and veins were both negative for lectin binding. However, these larger vessels were easily visualized without the lectin, and their walls tended to be autofluorescent.

Immunostaining for Factor VIII-R antigen in cryostat sections of rat muscle 


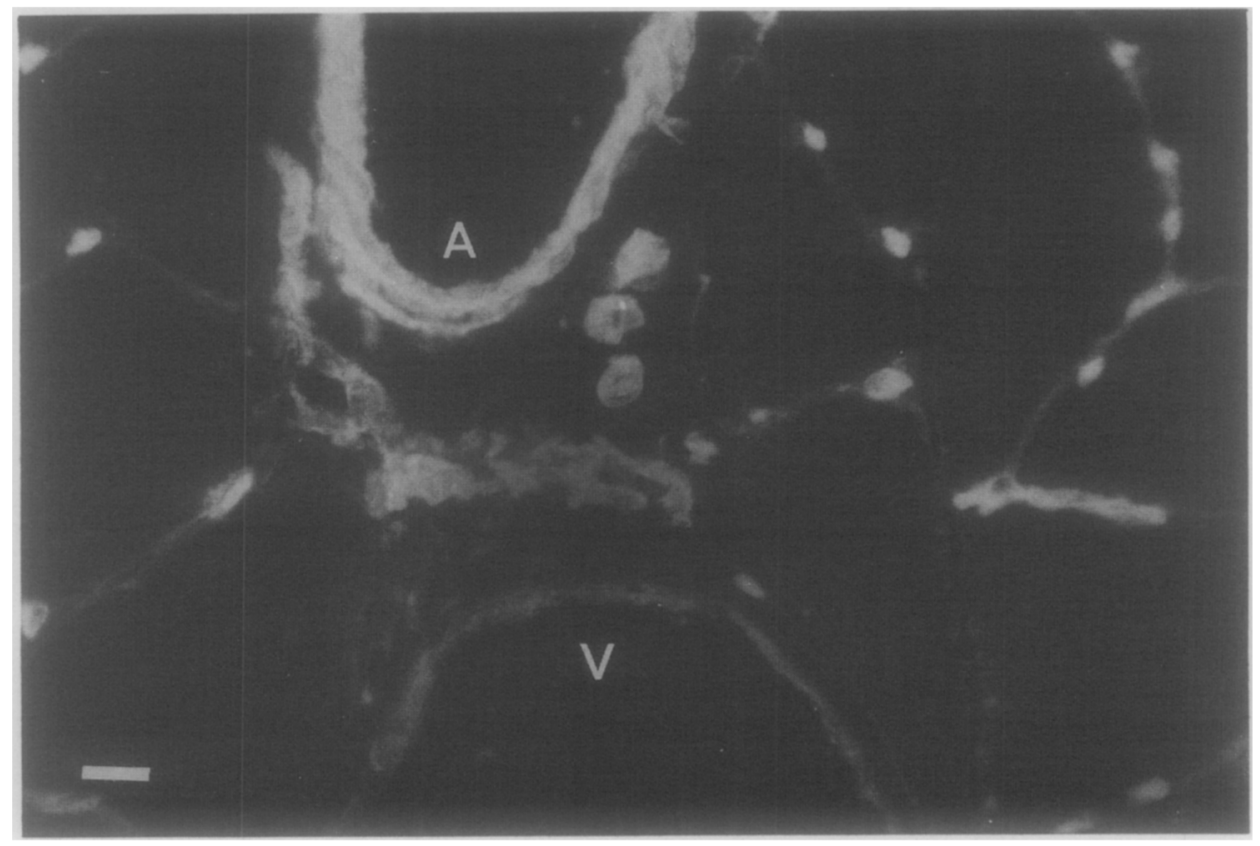

FIG. 4. Fluorescence due to FITC-GSI-B ${ }_{4}$ binding is intense around capillaries and small arteries (A) but relatively weak around veins (V).

revealed that the distribution of GSI binding is considerably more extensive than that of the VIII-R. The capillaries around the muscle fibers were essentially negative for the VIII-R antigen. Immunostaining was only occasionally detectable in the capillary bed using immersion objectives, and even then was minimal. In contrast, a granular distribution of VIII-R antigen was associated with the endothelium of arterioles, arteries, veins, and venules, but not their smooth muscle. An exact correspondence was observed between these vessels and the GSI binding (Fig. 8). Many vessels larger than 8-10 $\mu \mathrm{m}$ were at least marginally positive for Factor VIII-R. However, the density of the VIII-R associated with the venous endothelium was consistently greater than that associated with the arterioles and arteries.

In the GSI/APase double-staining experiment, comparisons were made of the sections that were stained individually and those that were double-stained. The GSI fluorescence was markedly reduced overall by the APase staining. Wherever APase was present, the GSI binding was almost completely obliterated (Fig. 9). Where faint staining for alkaline phosphatase occurred, some faint GSI binding could also be detected. Only those structures which showed no APase reaction product whatsoever were easily visible, but the fluorescence was nevertheless significantly dimmer. This was not due to the fixation, since the same problem occurred when unfixed sections were reacted for APase. Typical exposure time

FIG. 5. High magnification view of TRITC-GSI binding in rat cremaster muscle demonstrates the typical appearances of capillaries, arterioles, and venules in whole mount preparations of thin muscles. (A) Arcade of capillaries and venous capillaries; (B) terminal branching of arteriole; (C) capillary joining venule $(\operatorname{Bar}=10 \mu \mathrm{m})$. 

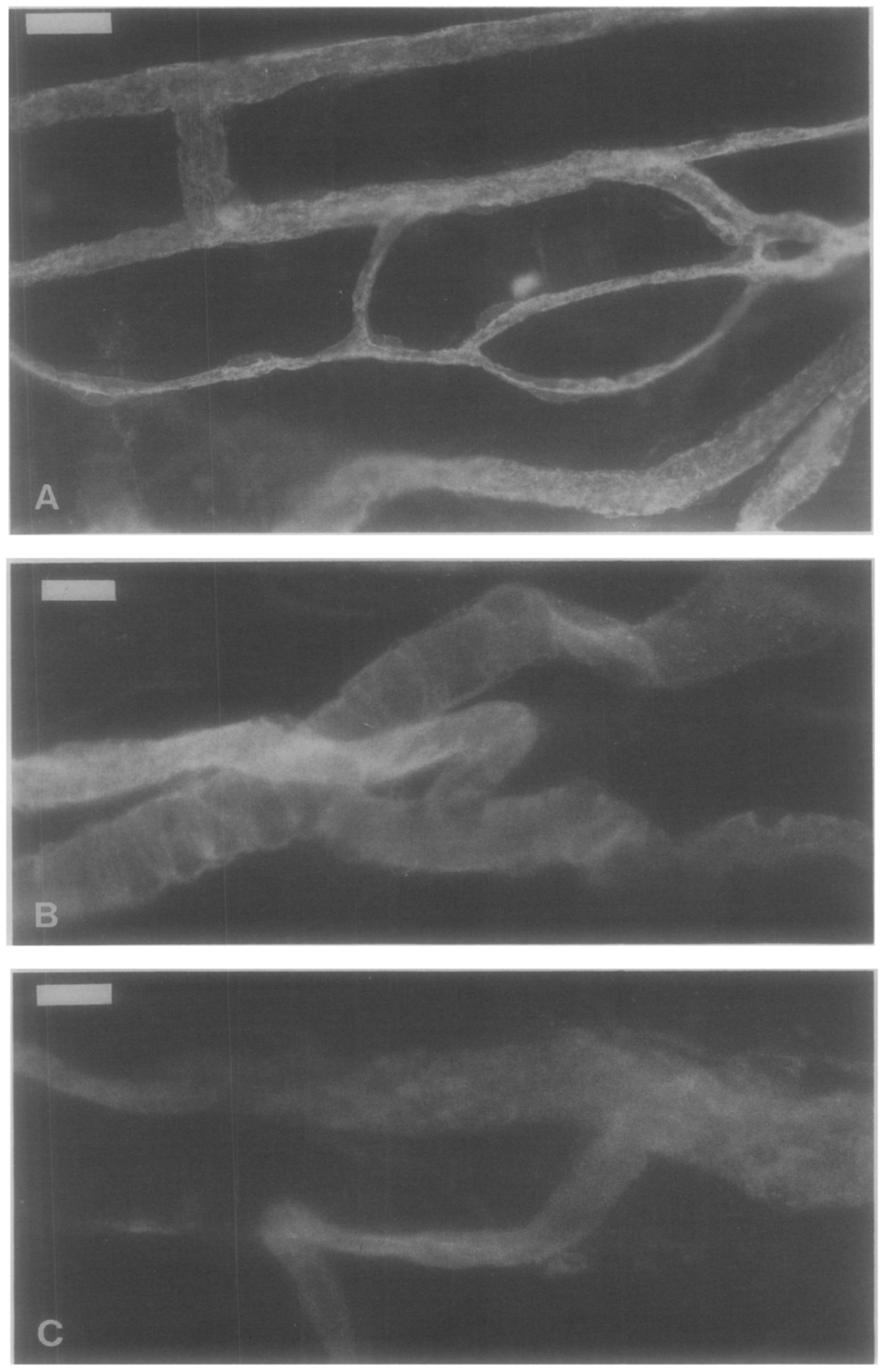


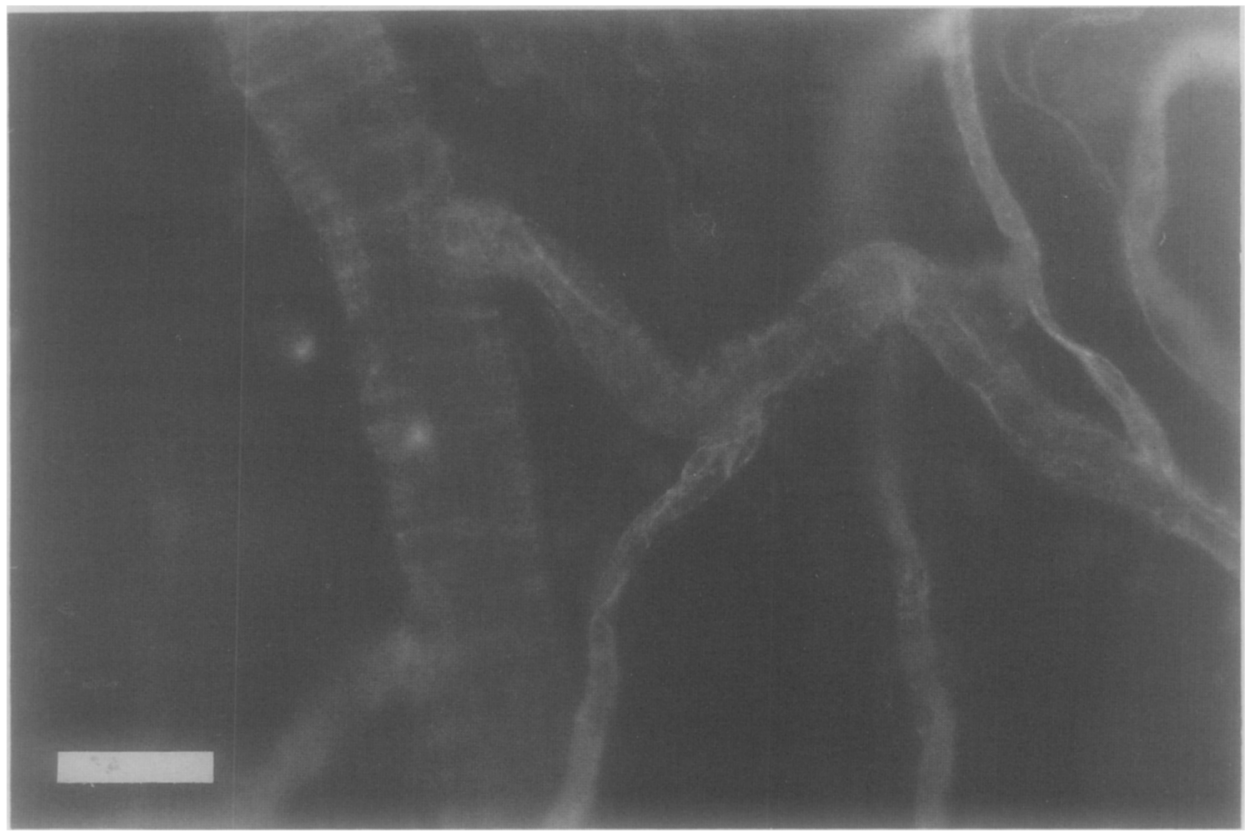

FIG. 6. TRITC-GSI binding highlights circular arrangement of smooth muscle cells in terminal branches of arterioles in hamster cheek pouch preparation. Note increased intensity of fluorescence in capillaries compared to arterioles. $($ Bar $=10 \mu \mathrm{m}$ ).

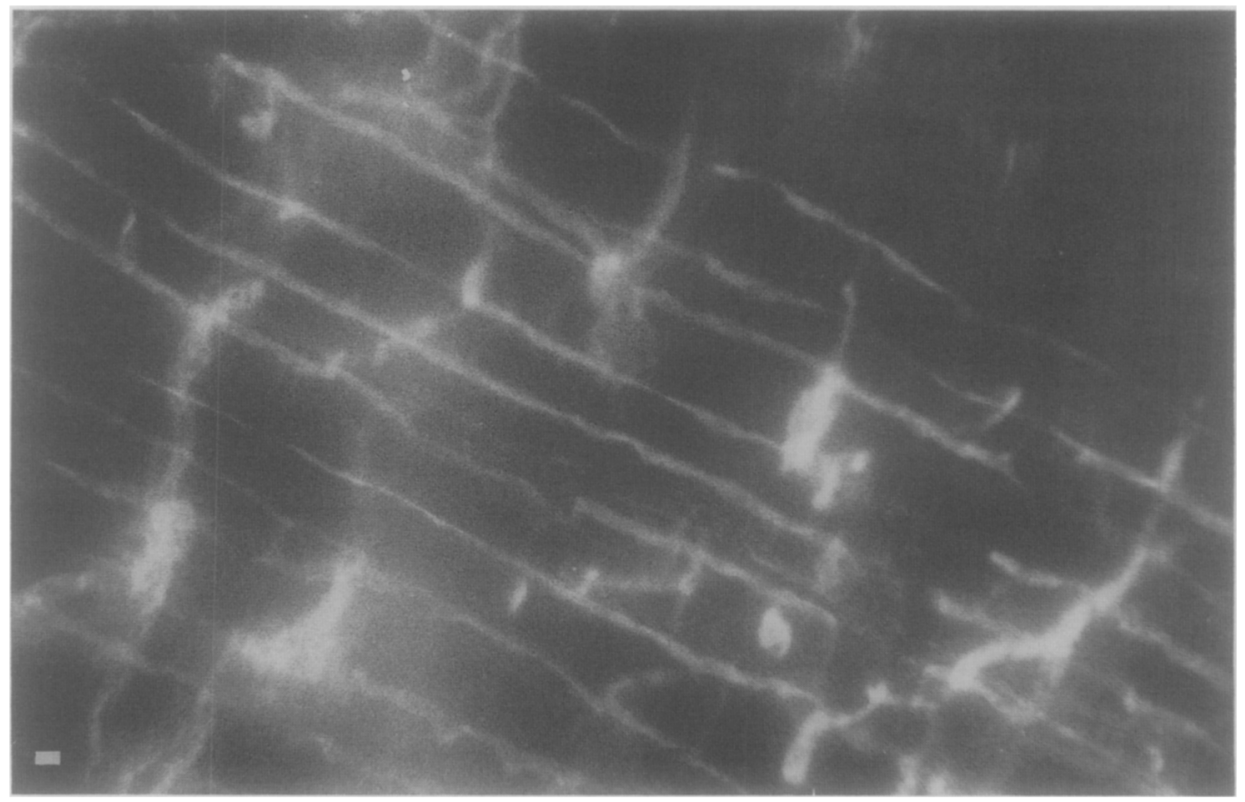

FIG. 7. Whole mount of unfixed mouse diaphragm reveals FITC-GSI-B ${ }_{4}$ binding in capillaries $($ Bar $=20 \mu \mathrm{m})$. 

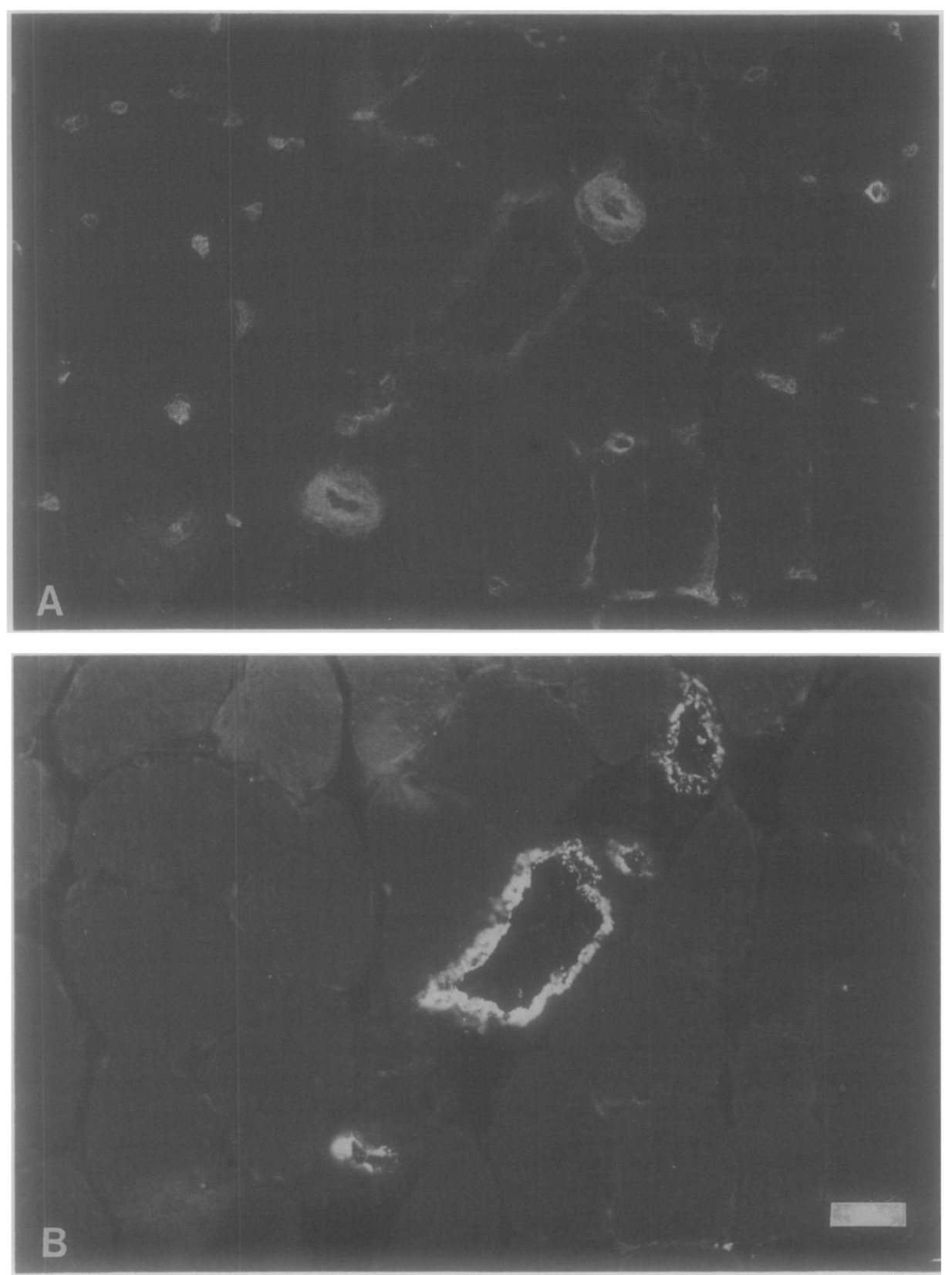

FIG. 8. Comparison between distribution of microcirculatory vessels as revealed by TRITC-GSI (A) and anti-Factor VIII-R (B). Capillaries and arterioles are strongly fluorescent with GSI, whereas most capillaries are negative for anti-Factor VIII-R, and arterioles are relatively weakly labeled. In contrast, veins are intensely labeled by VIII-R, but bind little GSI.

for photography of the GSI alone was 8-10 sec, whereas that for GSI when double-labeled with AP was 80-100 sec at an ASA setting of 400 .

Sections of rat sternomastoid, soleus, and extensor digitorum longus muscle that were double-stained for R-GSI binding and APase activity revealed a marked 

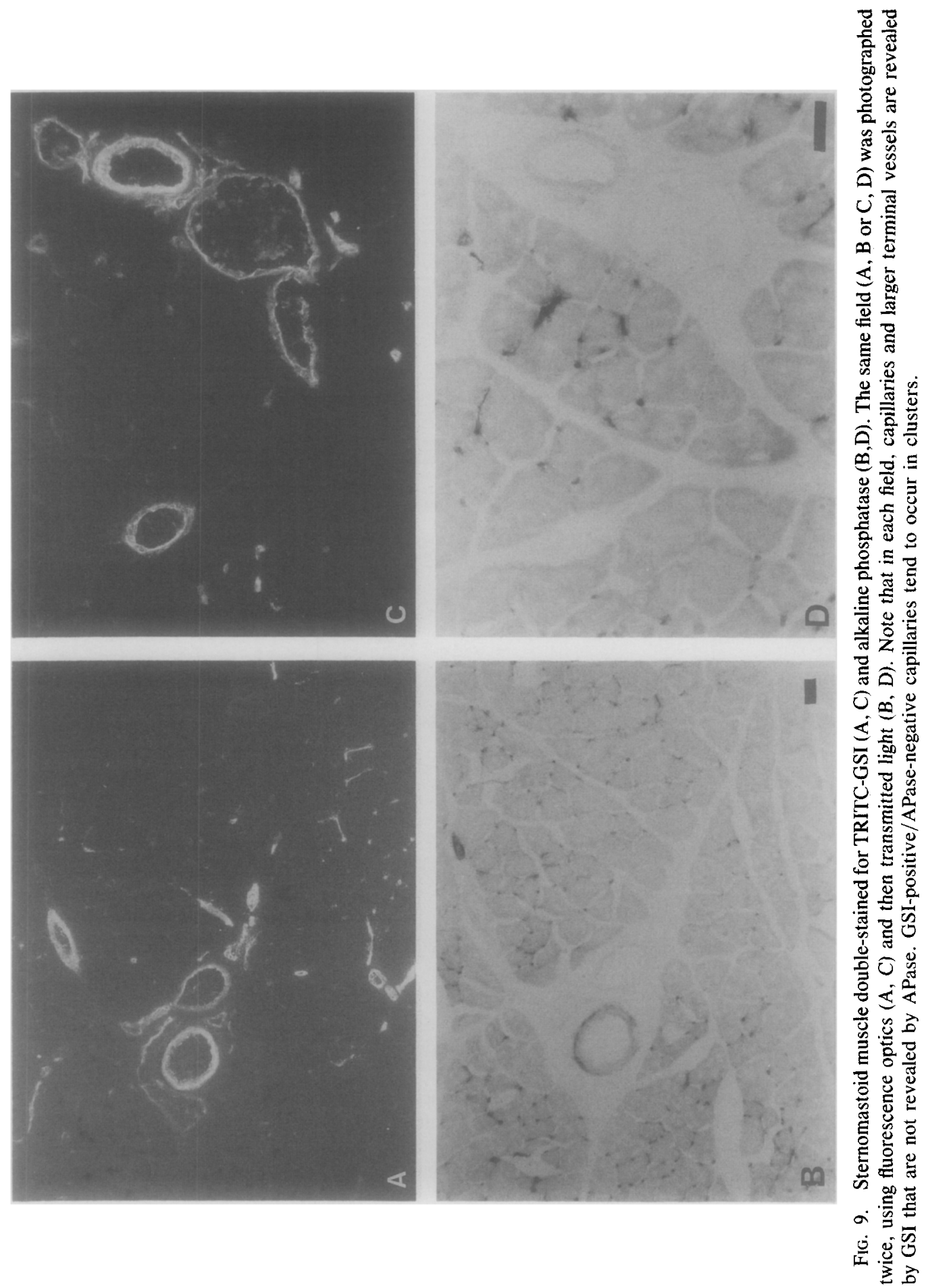
TABLE 2

Co-localization of Griffonia simplicifolia I Binding and Alkaline Phosphatase Activity in Rat Sternomastoid Muscle

\begin{tabular}{|c|c|c|c|}
\hline APase $^{a}$ & $\mathrm{GSI}^{a}$ & Red region $(\% \pm \mathrm{SEM})^{b}$ & White region $(\% \pm S E M)^{b}$ \\
\hline+ & - & $72.7 \pm 3.3$ & $52.5 \pm 3.7$ \\
\hline - & + & $27.3+3.3$ & $47.4 \pm 4.0$ \\
\hline
\end{tabular}

a A single $12-\mu \mathrm{m}$ cryostat section was exposed to TRITC-GSI and then reacted for APase, as described in the text. (+ indicates presence of APase staining or GSI fluorescence; - indicates lack of detectable APasc or fluorescence). No attempt was made to differentiate the samples according to the intensity of the enzyme reaction product or the fluorescence.

${ }^{b}$ The sternomastoid muscles were examined from four $250 \mathrm{~g}$ Male Sprague-Dawley rats. Three fields each in the red and white regions of the muscle were photographed. Each field was photographed twice, using epifluorescent illumination for TRITC-GSI, then using transmitted illumination for APase. The total number of APase or GSI-positive capillaries was tallied for each muscle $(n=185,227$, 232,201 capillaries per field in the red region; $n=79,117,113,93$ capillaries per field for the white region. The means $\pm \mathrm{SE}$ are calculated from the mean percentages of the total capillary count per muscle for $n=4$ muscles.

difference between the two endothelial markers. This was quantitated in the sternomastoid muscles (Table 2). A significant number of capillaries (and possibly venules) were APase-negative but GSI-positive in the double-stained sections. This was particularly striking in the sternomastoid muscle, where large fields of the muscle failed to show APase associated with the capillaries (Fig. 9). The sternomastoid muscle can be visually divided into a red region and a white region, which run parallel for the length of the muscle. Although the regional variation was most pronounced in the white region of the muscle, a significant number of APase-negative capillaries were also found within the red region, although they were in less noticeable clusters. Some capillaries showed reactivity for both APase and GSI binding, although the GSI fluorescence was invariably very weak. Because the GSI binding of strongly AP capillaries was obliterated, comparisons were made against some of the singly stained adjacent sections to determine whether there were any APase-positive capillaries that failed to bind GSI. None were found. Many terminal arterioles and venules that were negative for APase activity were revealed with the GSI method. However, we found no examples of APase-positive vessels that were negative for GSI.

\section{DISCUSSION}

Selective binding of certain lectins to microvascular elements has previously been noted in the literature (Peters and Goldstein, 1979; Pena et al., 1981; Holthofer et al., 1982; Gerhart, 1986; Minimikawa et al., 1987; Laitenen, 1987; Ribera et al., 1987). However, the use of the lectins in this context has been limited to the characterization of surface molecules (i.e., Simionescu and Simionescu, 1986). There are presently few published applications of this technique to other types of physiological problems. On the basis of observations in this laboratory and others (Hansen-Smith, 1985; Peters and Goldstein, 1979; Laitenen, 1987), we postulated that the GSI lectin or the more specific GSI-B ${ }_{4}$ isomer would be suitable as a fluorescent marker for physiological studies of the mi- 
crovascular bed of skeletal muscle. We therefore examined GSI-B 4 binding in a number of commonly used mammalian species in order to evaluate whether this marker had a widespread general application. Muscles from both the extremities and the trunk were examined. Similar results were obtained for mice, rats, hamsters, rabbits, dogs, and monkeys, suggesting that the techniques tested here have broad application for physiological studies. The samples of dog and monkey muscle did not, however, contain enough of the terminal vessels to determine whether the observations extended to structures other than capillaries in the large laboratory animals. It should be noted that the GSI lectin is blood-group specific in humans. Thus, the application of this lectin for human studies is limited to subjects having the B blood group (Capaldi et al., 1985, Hansen-Smith and McCoy, unpublished). In this respect, the GSI lectin differs from the Ulex lectin, which is not blood-group specific in humans.

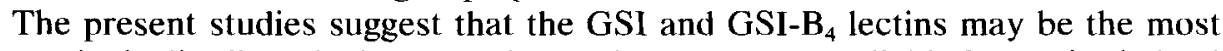
extensively distributed microvascular marker presently available for use in skeletal muscle. Previous reports noting the specificity of GSI binding to endothelium in muscle have only reported its association with capillary endothelium. However, our GSI binding studies revealed an entire physiological category of vessels, i.e., the terminal arterioles and venules of the vascular bed. Many of these are undetectable in cryostat sections using the APase method. Thus, GSI binding may be an extremely useful histochemical marker in studies of the distribution of terminal vessels of muscles that are too thick to be examined by intravital microscopy.

Our double-labeling experiments with VIII-R and APase both revealed a far more extensive microvascular bed with GSI than could be demonstrated with either of the other markers. The antibody we employed failed to reliably detect Factor VIII-R antigen in muscle capillaries, whereas GSI binding to capillaries was extensive. There was an apparent inverse relationship between the expression of GSI binding sites and Factor VIII-R antigen by the arteries and arterioles vs. venules and veins. We interpret this as a real difference in the concentration of VIII-R rather than an apparent increase that might result from folding of the walls of veins. This combination of markers may thus be useful in identifying regional changes in future studies.

This study represents one of the few in which the validity of a probe as a marker for capillaries has been evaluated by an independent method. The present results strongly suggest the GSI lectin binding is more suitable than APase activity when the latter is used by itself. APase-negative capillaries were sometimes clustered. Presumably, these represent the capillaries that become evident when PAS or diaminopeptidase activity is used in combination with APase (Mrazkkokova et al., 1986; Ogilvie and DeHay, 1986). It has been suggested that these APasenegative capillaries represent venular capillaries (Lojda, 1979). Since GSI is thought to bind the capillary basal laminae, (Peters and Goldstein, 1979), GSI may give results comparable to those obtained when PAS is used to detect the basal laminae of endothelial cells that are not reactive for APase. The advantage of GSI over PAS, however, is that there is no binding of the lectin to the basal lamina of the muscle fiber, and the sections can be analyzed at a lower magnification.

The GSI lectin is clearly an appropriate histochemical marker for both capillaries and other microvascular elements in muscle. Several other considerations favor its adoption for many of the histological approaches to microvascular questions. 
A practical advantage of using the lectin binding method over that detecting enzyme activity is that the sections need not be processed immediately. The GSI lectin binding sites are extremely stable during prolonged storage. A further advantage is that the GSI lectin may be used either as a fluorescent derivative or as a derivative such as peroxidase, that can be examined by transmitted light microscopy. However, in the latter application, precautions must be taken to control for the endogenous peroxidase activity of the erythrocytes. In our hands, the peroxidase method has given less satisfactory results than the fluorescent method and is therefore less preferable when both options are available. It should be noted, however, that the peroxidase derivative of the GSI lectin has been used on some fixed, paraffin-embedded tissues (Kuhlman and Peschke, 1984; Laitinen, 1987) which constitutes a further potential advantage of using the GSI lectin when transmitted light microscopy is necessary. Finally, unlike the APase reaction, neither the fluorescent derivatives nor the peroxidase derivative of GSI obscures the morphology of the capillaries, making possible measurements of capillary diameters.

Our observations of GSI binding to whole mounts of thin muscles indicate that the lectin may be extremely useful in delineating the terminal microvascular beds for intravital studies. The entire length of terminal vessels appears to be positive for the lectin binding. This was shown by following individual branches in the hamster cheek pouch and the rat cremaster muscles, and by focusing through the entire depth of field. Evidence from the whole mounts, combined with our observations in sectioned muscle, strongly suggest that most, if not all, of the terminal microvascular vessels bind the GSI lectin. Although the complete branching structure was not followed in these whole mounts because of the need to cut the muscles for mounting, we would classify the vessels that are visualized with GSI as fourth-order branching vessels.

For anatomical or vascular network studies this method has advantages which are not available from other methods. The function(s) of the terminal $\alpha$-gals to which the GSI and GSI-B 4 bind is presently unknown. It is assumed that the GSI binding will not interfere with the physiological functions of the microvascular bed, although this assumption remains to be tested. Preliminary studies in this laboratory have failed to reveal any toxic effect of the GSI lectin when applied in vivo. Minimikawa et al. (1987) have also recently suggested an in vivo application for this lectin, and they failed to note any toxicity of the lectins. However, the latter study, the lectin was applied by perfusion, whereas the application proposed in the present study does not require perfusion of the fluorescent marker.

The GSI lectin may be used advantageously in combination with other fluorescent vascular probes for intravital studies (Damon and Duling, 1984; Kayar and Banchero, 1985; Ley and Arfors, 1986). Previously it has only been possible to analyze those vessels which are being perfused (Tyml, 1986). Therefore, it has been necessary to deduce information about the size of the total microvascular bed by indirect measurements. Although the calculated total densities of capillary beds differ substantially from study to study, such calculations invariably suggest that only a fraction of the true capillaries are perfused at a given time (McDonagh et al., 1982; Damon and Duling, 1984). Utilization of the GSI lectin to delineate the microvascular bed, including capillaries, thus provides a potential method of testing the previous hypothetical conclusions.

In summary, these studies show that endothelial cells and smooth muscle cells 
from several species of mammals bind the GSI and GSI-B B $_{4}$ lectins. Differential binding is observed between the arterial and venous side, with a marked reduction of binding sites in the latter. GSI binding reveals significantly more capillaries and terminal arterioles than a histochemical technique such as APase activity. The property of GSI binding by the microvascular bed has potentially valuable applications for many physiological studies, including intravital studies of the microcirculation in thin skeletal muscles. The ability to visualize the microvascular bed by fluorescence microscopy will significantly enhance the ability of investigators to interpret results using other types of fluorescent probes. Among the possible histological and/or in vitro applications are double-labeling experiments with antibodies to other microvascular structures, hormones, and receptors, as well as using the lectin in combination with nonimmunological fluorescent techniques such as those used for visualizing neurotransmitters.

\section{ACKNOWLEDGMENTS}

The authors are indebted to Dr. Julian Lombard for his helpful comments during the preparation of this manuscript. Drs. I. D'Alecy, D. Carlson, and M. V. Riley kindly provided tissues from the dogs, monkey, and rabbits. The technical assistance of Jill Rheinheimer, Carole Brangwyn, and Michelle Tulak is gratefully acknowledged. This article is dedicated to the memory of Carole Brangwyn. This work was supported by grants from the American Heart Association of Michigan and the National Institutes of Health (NS-DE07826-01 and GM29470).

\section{REFERENCES}

Capaldi, J. M., Dunn, M. J., Sewry, C. A., and Dubowitz, V. (1985). Lectin binding in human skeletal muscle: A comparison of 15 different lectins. Histochem. J. 17, 81-92.

Damon, D. H., AND Duling, B. R. (1984). Distribution of capillary blood flow in the microcirculation of the hamster: An in vivo study using epifluorescent microscopy. Microvasc. Res. 27, 81-95.

Dawson, J. M., Tyler, K. R., And Hudlicka, O. (1987). A comparison of the microcirculation in rat fast glycolytic and slow oxidative muscles at rest and during contractions. Microvasc. Res. $33,167-182$.

Gerhart, D. Z., Zl.onis, M. S., AND Drewes, L. R. (1986). Light and electron microscopic localization of D-Galactosyl residues in capillary endothelial cells of the canine cerebral cortex. J. Histochem. Cytochem. 641-648.

Graham, R. C., AND Karnovsky, M. J. (1966). The early stages of absorption of injected horseradish peroxidase in the proximal tubules of mouse kidney. Ultrastructural cytochemistry by a new technique. J. Histochem. Cytochem. 14, 291-302.

Hansen-Smith, F. M. (1985). Differences between the composition of muscle fiber and capillary basal laminae. Anat. Rec. 211, 78A.

HoLTHOFER, H. (1983). Lectin binding sites in kidney: A comparative study of 14 animal species. $J$. Histochem. Cytochem. 31, 531-537.

Hoi.thofer, H., Virtanen, I., Kariniemi, A. I., Hormia, M., I.indfr, F., ann Mifttinfa, A. (1982). Ulex europaeus I lectin as a marker for vascular endothelium in human tissues. Lab. Invest. 47, $60-66$.

Huduicka, O. (1982). Growth of capillaries in skeletal and cardiac muscle. Circ. Res. 50, 451-460.

Kayar, S. R., and Banchero, N. (1985). Sequential perfusion of skeletal muscle capillaries. Microvasc. Res. 30, 298-305.

Kuhlman, W. I., And Peshre, P. (1984). A comparative study of procedures for histological detection of lectin binding by use of Griffonia simplicifolia agglutinin I and gastrointestinal mucosa of the rat. Histochemistry 81, 265-272.

LaTinen, L. (1987). Griffonia simplicifolia lectins bind specifically to endothelial cells and some epithelial cells in mouse tissues. Inistochem. J. 19, 225-234.

LeY, K., AND ARfors, K. E. (1986). Segmental differences of microvascular permeability for FITCdextrans measured in the hamster cheek pouch. Microvasc. Res. 31, 84-99. 
LIPENSKA, M., AND HoRSKy, J. (1984). Distribution of capillary bed segments with alkaline phosphatase and dipeptidylaminopeptidase IV activity in skeletal muscle of the rat. Folia Morphol. 32, 69-76.

LOJDA, Z. (1979). Studies on dipeptidyl (amino) peptidase IV (glyclyl-proline napthylamidase) II. Blood vessels. J. Histochem. 17, 555-557.

Maxwell, L. C., White, T. P., and Faulkner, J. A. (1980). Oxidative capacity, blood flow, and capillarity of skeletal muscles. J. Appl. Physiol. 49, 627-633.

McCoy, J. P. (1986). Contemporary laboratory applications of lectins. Biotechniques 4, 252-262.

McDonagh, P. F., Gore, R. W., And Gray, S. D. (1982). Perfused capillary surface area in postural and locomotor skeletal muscle. Microvasc. Res. 24, 142-157.

Minimikawa, T., Miyake, T., Takamutsu, T., and Fujita, S. (1987). A new method of lectin histochemistry for the study of brain angiogenesis. Lectin angiography. Histochemistry 87, 317320 .

Mrazkrokova, O., Grim, M., and Carlson, B. (1986). Enzymatic heterogeneity of the capillary bed of rat skeletal muscles. Amer. J. Anat. 177, 141-148.

Murphy, L. A., and Goldstein, I. J. (1977). Five $\alpha$-D-galactopyranosyl-binding isolectins from Bandeiraea simplicifolia seeds. J. Biol. Chem. 252, 4739-4742.

OGILvie, R. W., and Dehay, P. L. (1986). Histochemical demonstration of skeletal muscle capillaries by combining stain for alkaline phosphatase and glycoproteins Anat. Rec. 214, 92A.

Peters, B. P., AND Goldstein, I. J. (1979). The use of fluorescein-conjugated Bandeiraea simplicifolia $\mathrm{B}_{4}$-isolectin as a histochemical reagent for the detection of $\alpha$-D-galactopyranosyl groups: Their occurrence in basement membranes. Exp. Cell Res. 120, 321-334.

Plyley, M. J., AND Groom, A. C. (1975). Geometrical distribution of capillaries in mammalian straited muscle. Amer. J. Physiol. 228, 1376-1383.

Ponder, B. A. J., Festing, M. F. W., and Wilkinson, M. M. (1985). An allelic difference determines reciprocal patterns of expression of binding sites for Dolichos biflorus lectin in inbred strains of mice. J. Embryol. Exp. Morphol. 87, 229-239.

Ponder, B. A. J., AND Wilkinson, M. M. (1983). Organ-related differences in binding of Dolichos biflorus agglutinin to vascular endothelium. Dev. Biol. 96, 535-541.

Potter, R. F., AND Groom, A. C. (1983). Capillary diameter and geometry in cardiac and skeletal muscle studied by means of corrosion casts. Microvasc. Res. 25, 68-84.

Ribera, J., Esquerda, J. E., and Comella, J. X. (1987). Phylogenetic polymorphism on lectin binding to junctional and non-junctional basal lamina at the vertebrate neuromuscular junction. Histochemistry 87, 301-307.

Romanul, F. D. A., and Bannisiek, G. (1962). Localized areas of high alkaline phosphatase activity in the terminal arterial tree. J. Cell Biol. 15, 73-84.

Schraufnagel, D. E., Roussos, C., Macklem, P. T., and Wang, N. S. (1983). The geometry of the microvascular bed of the diaphragm: Comparison to the intercostals and triceps. Microvasc. Res. 26, 291-306.

Sillau, A. H., and Banchero, H. (1977). Visualization of capillaries in skeletal muscle by the ATPase reaction. Pfleugers Arch. 369, 269-271.

Simionescu, M. and Simionescu, N. (1986). Functions of the endothelial cell surface. Annu. Rev. Physiol. 48, 279-293.

Simon, R. H., McCoy, J. P., Chu, A. E., Dehart, P. D., and Goldstein, I. J. (1986). Binding of Griffonia simplicifolia I lectin to rat pulmonary alveolar macrophages and its use in purifying type II alveolar epithelial cells. Biochim. Biophys. Acta 885, 34-42.

TYмь, K. (1986). Capillary recruitment and heterogeneity of microvascular flow in skeletal muscle before and after contraction. Microvasc. Res. 32, 84-98.

VAN Reempts, J., Haseldonck, M., ANd Borgers, M. (1983). Microvascular geometry and tissue perfusion, allowing simultaneous histopathologic evaluation. Microvasc. Res. 25, 300-306.

VetTerlein, F., AND Schmidt, G. (1983). Histological demonstration of capillaries, interstitial space and muscle fibers in heart and skeletal muscle with fluorescent dyes. Acta Anat. 116, 285-89. 